\title{
Decreased activation-induced cell death by EBV-transformed B-cells from a patient with autoimmune lymphoproliferative syndrome caused by a novel FASLG mutation
}

\author{
Raquel Ruiz-García1, Sergio Mora1', Gema Lozano-Sánchez², Luis Martínez-Lostao³, Estela Paz-Artal1,4,5 , Jesús Ruiz-Contreras4, \\ Alberto Anel ${ }^{3}$, Luis I. González-Granado ${ }^{4,6}$, David Moreno-Pérez² and Luis M. Allende ${ }^{1,4}$
}

BACKGROUND: Autoimmune lymphoproliferative syndrome (ALPS) is a primary immunodeficiency characterized by chronic lymphoproliferation, autoimmune manifestations, expansion of double-negative T-cells, and susceptibility to malignancies. Most cases of ALPS are caused by germline or somatic FAS mutations. We report the case of an ALPS patient due to a novel homozygous Fasligand gene mutation (ALPS-FASLG).

METHODS: ALPS biomarkers were measured and FASLG mutation was identified. Functional characterization was carried out based on activation-induced cell death (AICD) and cytotoxicity assays.

RESULTS: This report describes the cases of a patient who presented a severe form of ALPS-FASLG, and his brother who had died due to complications related to ALPS. Moreover, in another family, we present the first case of lymphoma in a patient with ALPS-FASLG. Functional studies showed defective Fasligand-mediated apoptosis, cytotoxicity, and AICD in T-cell blasts. Otherwise, expression of the FASLG gene and corresponding protein was normal, but the shedding of the Fasligand was impaired in T-cells. Additionally, analyzing Epstein-Barr virus (EBV)-transformed B-cells, our results indicate impaired AICD in ALPS-FASLG patients.

CONCLUSION: Patients with autosomal recessive inheritance of ALPS-FASLG have a severe phenotype and a partial defect in AICD in T- and B-cell lines. The Fasligand could play a key role in immune surveillance preventing malignancy.

Cinically, patients with autoimmune lymphoproliferative syndrome (ALPS) have nonmalignant lymphadenopathies, splenomegaly, autoimmune cytopenias, and susceptibility to malignancy (1-5). Immunological features of ALPS consist of an expanded population of double-negative (DN) T-cells (TcR $\alpha \beta+C D 4-C D 8-)$, while elevated levels of circulating plasma biomarkers, including IL-10, IL-18, soluble Fas ligand (sFasL), and vitamin B12, have also been described
$(6,7)$. ALPS patients are classified according to the underlying genetic defect (Mendelian inheritance in man (MIM) no. 601859). Most cases of ALPS are associated with germline (ALPS-FAS) or somatic (ALPS-sFAS) heterozygous FAS mutations or a combination of both $(3,8,9)$. Mutations in the Fas ligand gene (FASLG) in ALPS (ALPS-FASLG) have only been reported in five patients, two of them with the autosomal dominant form $(10,11)$ and three with the autosomal recessive form of the disease (12-14). Cases with clinical and immunological features of ALPS with unknown genetic defects are referred to as ALPS-U (15). In addition, the demonstration of an apoptosis defect is critical for the diagnosis of ALPS. Specifically, activation-induced cell death (AICD), a homeostatic mechanism involved in the termination of the adaptive immune response by extrinsic or intrinsic cell death pathways, is impaired in ALPS-FASLG patients $(12,13,16)$ (see Supplementary Table S1 online).

This report describes the cases of two Moroccan brothers (P1 and P2), born to consanguineous parents, who presented a severe form of ALPS caused by a novel homozygous FASLG mutation. Given that alterations in immune tolerance have been described in ALPS patients (3), we decided to assess the impact of Fas ligand (FasL) deficiency on cytotoxicity and apoptosis both in T-cell blasts and B-cell lines transformed with EpsteinBarr virus (EBV). These results are compared with those of a patient with FasL deficiency (P3) who, since previously being described by our group (12), has had a lymphoma.

\section{RESULTS}

Clinical, Immunologic, and Molecular Features of ALPS-FASLG Patients

At the age of $8 \mathrm{mo}$, P1 was admitted to hospital for persistent generalized lymphadenopathy and hepatosplenomegaly, thrombocytopenia and anemia, accompanied by intermittent fever and diarrhea. He also had upper respiratory tract infections, bronchiolitis, and pneumonia. The most prominent

\footnotetext{
'Servicio de Inmunología, Hospital Universitario 12 de Octubre, Madrid, Spain; ²UGC de Pediatría, Hospital Materno-Infantil, Hospital Regional Universitario, Málaga, Spain; ${ }^{3}$ Departamento de Bioquímica, Biología Molecular y Celular, Universidad de Zaragoza, Zaragoza, Spain; ${ }^{4}$ Instituto de Investigación I+12, Madrid, Spain; ${ }^{5}$ Facultad de Medicina, Universidad Complutense y Sección de Inmunología, Universidad San Pablo CEU, Madrid, Spain; ${ }^{6}$ Unidad de Inmunodeficiencias, Departamento de Pediatría, Hospital Universitario 12 de Octubre, Madrid, Spain. Correspondence: Luis M. Allende (luis.allende@salud.madrid.org) 
immunological abnormalities in P1 included increased percentage and number of DN T-cells, high plasma IL-10, vitamin B12 and sCD25 levels, and hypergammaglobulinemia. However, sFasL was not detected, unlike in ALPS-FAS patients (Table 1). Mutations in the FAS gene were also ruled out in this patient. At this stage, ALPS-FASLG was suspected.

FASLG sequencing analysis revealed a novel homozygous G-to-C substitution at cDNA nucleotide 605 (GenBank KJ948655), which produces a nonconservative mutation C202S in exon 4 affecting the extracellular domain of the protein. The mutation was confirmed in cDNA and genomic DNA. This mutation was not found as a single nucleotide polymorphism and was absent in 100 healthy donors. We found that codon 202

Table 1. Immunological features of patient P1

\begin{tabular}{|c|c|c|}
\hline & Normal range & Patient \\
\hline $\begin{array}{l}\text { Lymphocyte count (no./ } \mu \mathrm{l} \text { ) and } \\
\text { phenotype (\%) }\end{array}$ & $3,000-8,000$ & 5,634 \\
\hline \multicolumn{3}{|l|}{ T-cells } \\
\hline $\mathrm{CD} 3+(/ \mu \mathrm{l})$ & $1,800-5,500$ & 4,844 \\
\hline TCRaß (\%) & $55-75$ & 83 \\
\hline $\mathrm{TCR} \gamma \delta(\%)$ & $0-7$ & 1 \\
\hline Double-negative T-cells (\%) & $0-4$ & 8 \\
\hline $\mathrm{CD} 4+(/ \mu \mathrm{l})$ & $1,000-3,800$ & 2,707 \\
\hline $\mathrm{CD} 8+(/ \mu \mathrm{l})$ & $500-1,800$ & 1,698 \\
\hline \multicolumn{3}{|l|}{ Activated T-cells } \\
\hline CD3+HLA-DR+ (\%) & $0-10$ & 14 \\
\hline \multicolumn{3}{|l|}{ NK cells } \\
\hline 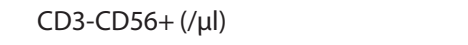 & $100-1,000$ & 169 \\
\hline iNKT Cells CD3+CD56+Vß11+Va24 (\%) & $0.01-1.80$ & 1.1 \\
\hline \multicolumn{3}{|l|}{ B-cells } \\
\hline 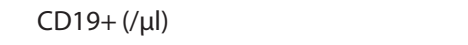 & $800-2,200$ & 507 \\
\hline CD19+CD27+ (\%) & $12-35$ & 4.1 \\
\hline CD19+lgD+CD27-(naive) (\%) & $57-84$ & 90.1 \\
\hline CD19+lgD+CD27+(marginal) (\%) & $4-12$ & 1.3 \\
\hline CD19+lgD-CD27+(switched) (\%) & $7-25$ & 3 \\
\hline \multicolumn{3}{|l|}{ Serum immunoglobulins } \\
\hline $\lg G(\mathrm{mg} / \mathrm{dl})$ & $330-1,160$ & 2,640 \\
\hline $\lg A(\mathrm{mg} / \mathrm{dl})$ & $10-100$ & 158 \\
\hline $\lg M(\mathrm{mg} / \mathrm{dl})$ & $40-170$ & 220 \\
\hline \multicolumn{3}{|l|}{ Specific antibodies } \\
\hline IgG vs. tetanus toxoid (IU/ml) & $>0.15$ & 1.42 \\
\hline \multicolumn{3}{|l|}{ ALPS biomarkers } \\
\hline IL-10 (pg/ml) & $0-20$ & 98 \\
\hline sCD25 (pg/ml) & $0-1,900$ & 19,360 \\
\hline sFasL (pg/ml) & $0-250$ & 0 \\
\hline Vitamin B12 (pg/ml) & $200-753$ & $>2,000$ \\
\hline \multicolumn{3}{|l|}{ Autoimmunity } \\
\hline Antinuclear antibodies & & $+(1 / 160)$ \\
\hline
\end{tabular}

ALPS, autoimmune lymphoproliferative syndrome; SFasL, soluble Fas ligand. is conserved in 10 species other than humans, which underlines the importance of this amino acid for proper functioning of the protein (see Supplementary Figure S1a online). Both parents and one sibling are heterozygous carriers, and they are healthy without any clinical evidence or history of ALPS (see Supplementary Figure S1b online). The patient was treated with corticosteroids and rapamycin, and this led to a remarkable and rapid reduction of the size of his spleen, liver, and lymph nodes. Lower numbers of DN T cells, normal levels of immunoglobulins, negative antinuclear antibodies, and normal platelet counts were observed after starting the treatment. $\mathrm{He}$ recovered from CMV pneumonitis with treatment with valganciclovir. Mild pulmonary toxicity secondary to rapamycin was suspected, and resolved after decreasing the daily dose.

His sibling, P2, previously died at $3 \mathrm{y}$ of age with dramatic failure to thrive, massive hepatosplenomegaly, respiratory insufficiency, pancytopenia, and increased levels of ferritin, vitamin B12 and immunoglobulins. The FASLG mutation in this patient (C202S) was confirmed in genomic DNA from a postmortem sample (see Supplementary Figure S1b online).

P3 is an ALPS-FASLG patient, followed-up clinically for more than 19 y (12), who developed lymphoma. She complained of a 3 -wk period of flu-like illness accompanied by asthenia and dizziness. On physical examination, splenomegaly was noted $(8 \mathrm{~cm}$ below left costal margin). Blood tests were normal. Abdominal ultrasound revealed spleen enlargement $(15 \mathrm{~cm})$ and a heterogeneous retroperitoneal mass $(18 \times 7 \times 8 \mathrm{~cm})$ with a cystic component on its lower edge, located between left psoas muscle and ipsilateral kidney. Whole-body CT showed scattered axillary and upper mediastinal lymphadenopathies (all less than $1 \mathrm{~cm}$ in diameter), and abdominal lymphadenopathies located in the peripancreatic mesentery. Dimensions of the abdominal (solid and slightly hypodense) mass were $16 \times 11 \times 10 \mathrm{~cm}$ (see Supplementary Figure S2 online). Histopathologic examination of the mass revealed atypical cells positive for $\mathrm{CD}^{+}, \mathrm{CD}_{4} 3^{+}$, and $\mathrm{CD}^{+}$, and negative for $\mathrm{CD}^{+}, \mathrm{CD}^{+}, \mathrm{CD}^{2} 5^{+}$, $\mathrm{CD} 30^{+}, \mathrm{CD} 56^{+}, \mathrm{CD} 79 \mathrm{a}^{+}$, and cyclin $\mathrm{D}^{+}$. Further, $40 \%$ of the blasts were positive for Ki-67. This was consistent with a diagnosis of peripheral T-cell lymphoma. She was treated with the standard chemotherapy regimen for non-Hodgkin lymphoma (CHOP), and has been asymptomatic for the last $8 \mathrm{y}$ without disease flares. At the time of writing, this patient is pregnant and has no apparent lymphoproliferative or autoimmune phenomena. We conclude that, as in ALPS-FAS patients, clinical follow-up in ALPS-FASLG patients is warranted due to the risk of malignant lymphoproliferative disorders.

\section{Impaired FasL-Mediated Cytotoxicity}

To explore whether the FasL in P1 had lost its cytotoxic activity, cytotoxicity assays were performed using phytohemagglutinin (PHA)-activated T-cell blasts from P1, P3, and two healthy controls. T-cell blasts from P1 (like those from P3) caused less cytolysis of Jurkat cells than T-cell blasts from controls, suggesting that the FasL from P1 was inactive (Figure 1a).

To explore FasL functional activity in P1, full-length cDNA alleles (FASLG wild-type from a healthy control and mutant 
a

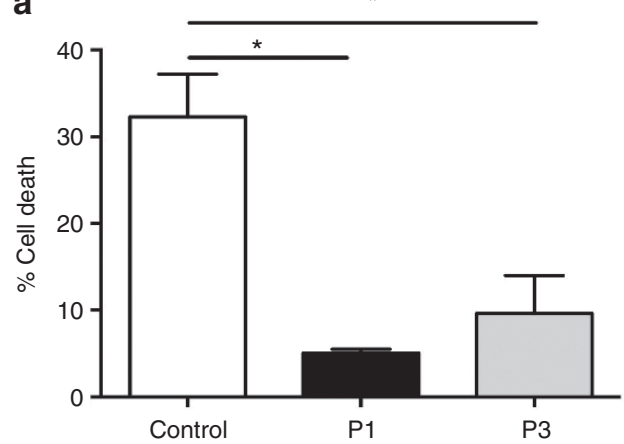

b

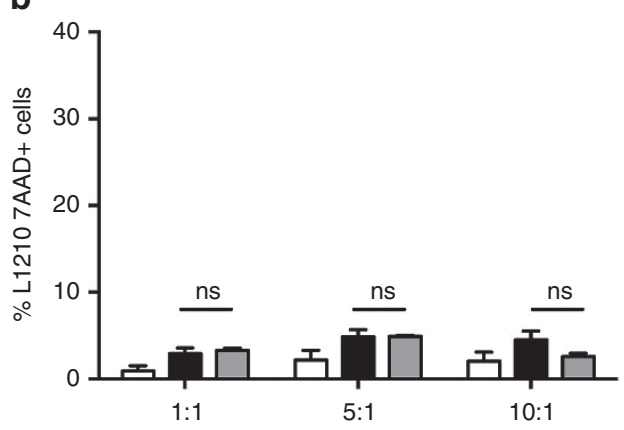

C
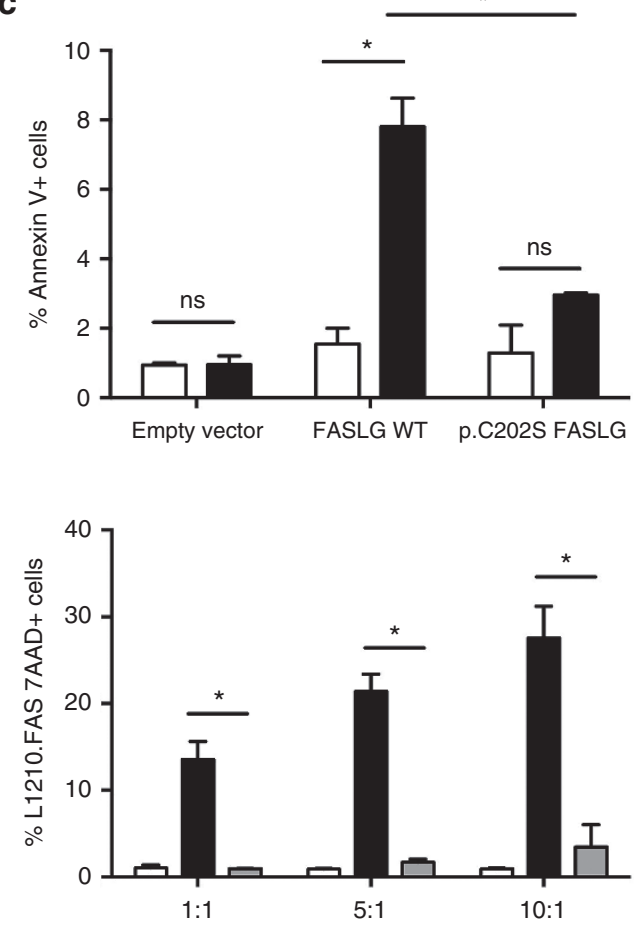

Figure 1. Functional studies of Fas/FasL-mediated cytotoxicity. (a) The graph represents the cytotoxicity of T-cell blasts from healthy controls ( $n=2$ ) and patients P1 and P3 on CFSE-stained Jurkat cells. (b) H293T cells were transfected with an empty vector $(\square)$, an expression vector containing the wildtype FASLG allele $(\square)$ or the mutated FASLG allele from patient P1 $(\square)$. Forty-eight hours after transfection, H293T cells were tested against CFSE-stained L1210 (left panel) and L1210.FAS (right panel) target cells at different effector:target ratios (as shown) in 6-h cytotoxicity assays. (c) Supernatants from the cultures assessed as indicated in (b) were collected and tested on L1210 ( $\square$ ) and L1210.FAS ( ) target cells. Columns represent the mean \pm SD of two independent experiments. Statistical comparisons were performed with unpaired Student's $t$-tests, and an asterisk indicates significance: ${ }^{*} P<0.05$.

from P1) were cloned into the pIRES2dsRED2 expression vector. These clones were then transfected into the effector cells (H293T) and cytotoxicity assays were performed using L1210. Fas and L1210 cells as targets. As shown by 7-AAD staining, H293T cells transfected with wild-type FASLG clearly induce death of L1210.Fas cells. In contrast, H293T cells transfected with mutant FASLG did not induce death in L1210.Fas target cells at effector:target (E:T) ratios of 1:1, 5:1, or 10:1. As a control, it was confirmed that no significant cytotoxicity was observed in Fas-negative L1210 cells under the same conditions (Figure 1b).

Additionally, the cytotoxic activity of FasL released on exosomes in H293T-transfected cells was measured in the supernatants using L1210.Fas cells as targets and L1210 cells as a negative control. Results showed that supernatants from the wild-type FASLG H293T-transfected cells induced a higher rate of death in L1210. Fas cells than supernatants from the mutant FASLG cells (Figure 1c).

\section{FasL Expression Analysis}

To rule out that the defective Fas-mediated cytotoxicity in T-cell blasts from P1 was caused by a low level of FasL expression, we analyzed expression of the FASLG gene and corresponding protein. The mRNA expression level, measured by RT-PCR analysis, was similar in P1 and in healthy controls, indicating that the FASLG mutation did not abrogate normal processing of the gene (Figure 2a). Additionally, the FasL protein was clearly detected in P1 T-cell blasts by western blot and the level of the protein was comparable to that in healthy controls (Figure 2b). Immunophenotypic analysis of both surface and cytoplasmic FasL expression of $\mathrm{CD}^{+}$and $\mathrm{CD}^{+} \mathrm{T}$ cells found comparable levels in P1, P3, and the healthy controls (data not shown). However, sFasL was not detectable in either plasma or cell supernatants of activated peripheral blood mononucleated cells (anti-CD3+anti-CD28 and PHA) from $\mathrm{P} 1$ and $\mathrm{P} 3$, unlike in those from healthy controls. These results indicate that the FASLG mutation in these patients inhibited the shedding of the sFasL protein (Figure 2c).

\section{Decreased Activation-Induced Cell Death}

The Fas/FasL pathway was evaluated using functional apoptosis assays. T-cell blasts from P1 showed normal Fas-mediated apoptosis when it was induced by an agonistic anti-Fas antibody (Apo1.3) (Figure 3a). However, we observed a partial reduction in AICD in T-cell blasts from P1 at all doses of PHA. T-cell blasts from a Fas-deficient patient (ALPS-FAS) were also used, and these showed impaired Fas-mediated apoptosis and conserved AICD. The AICD results in P1 were comparable with those previously observed in ALPS-FASLG patients $(12,13)$ (see Supplementary Table S1 online) (Figure $3 \mathbf{b}$ ).

However, our study indicates for the first time impaired AICD in ALPS-FASLG patients (P1 and P3) using EBV-transformed 


\section{Articles | Ruiz-García et al.}

a

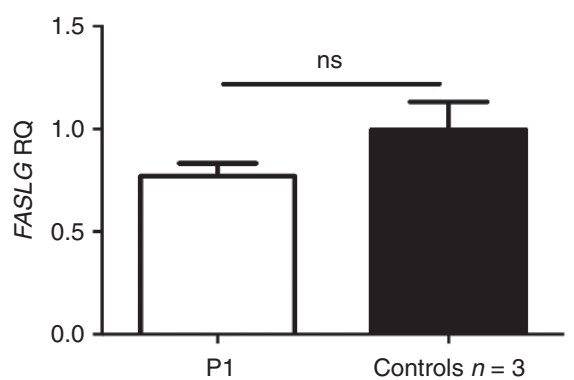

b

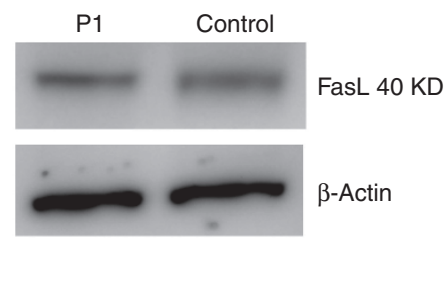

C

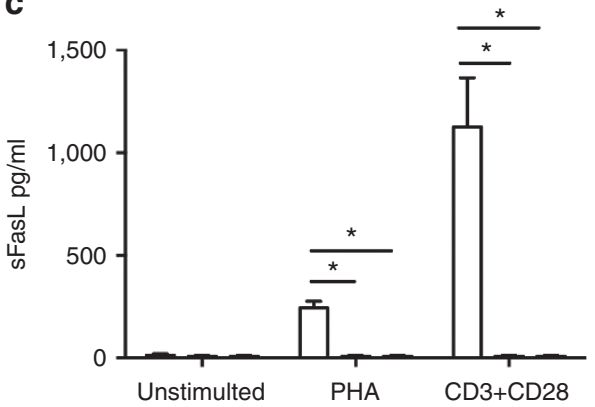

Figure 2. Expression of FasL. (a) FASLG gene expression in T-cell blasts from patient P1 and three healthy controls. (b) Western blot of FasL and $\beta$-actin protein expression from a healthy control and the patient. (c) Peripheral-blood mononuclear cells from controls $(\square)$, P1 ( $\square$ ) and P3 ( $\square$ ) were stimulated as indicated, after $72 \mathrm{~h}$, cell-free supernatants were harvested and sFasL measured as described in the Methods. Columns represent the mean \pm SD of two independent experiments. Statistical comparisons were performed with unpaired Student's $t$-tests, and an asterisk indicates significance: ${ }^{*} P<0.05$.

a

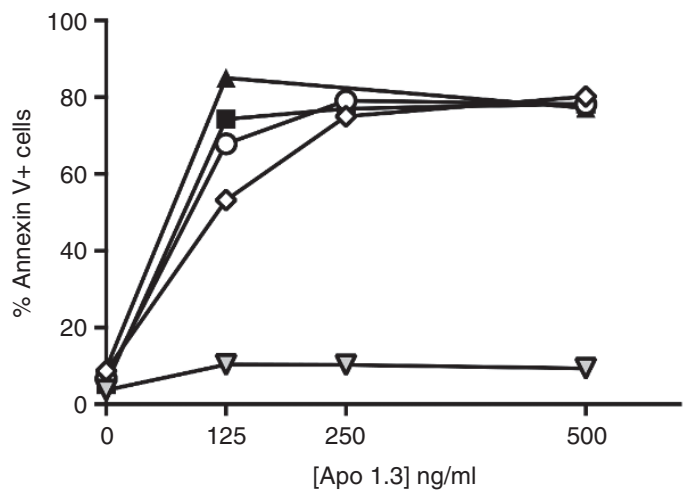

C

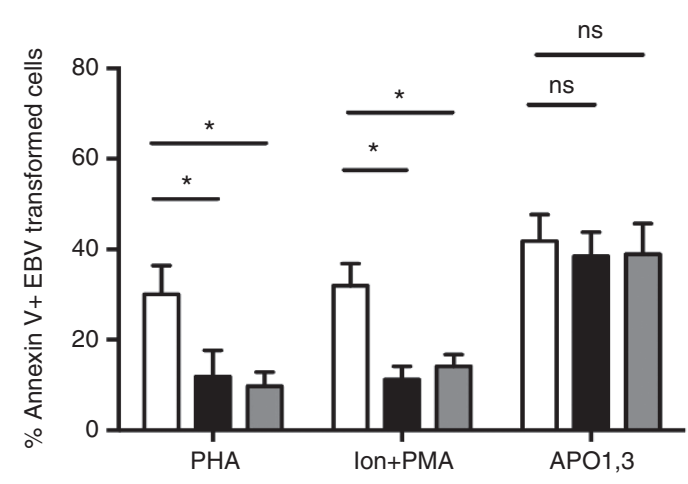

b

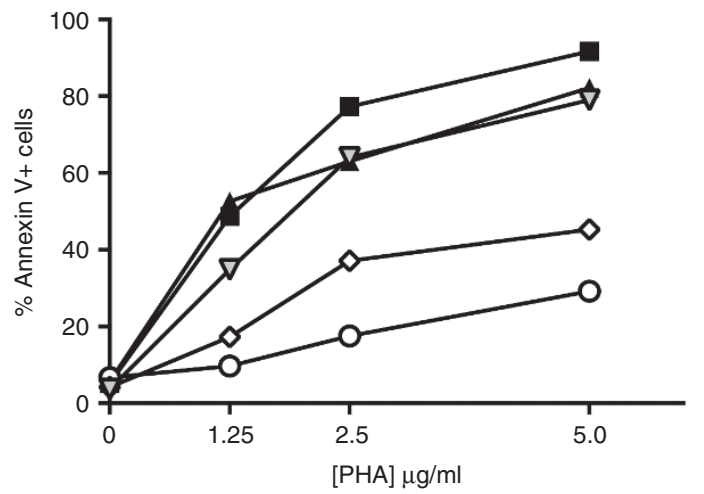

d

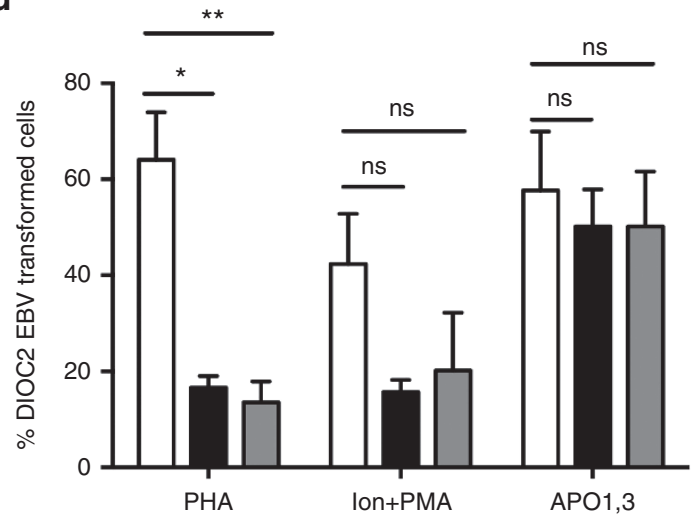

Figure 3. Fas-induced apoptosis and activation-induced cell death (AICD) in T-cell blasts and in EBV-transformed B-cells from patients P1 and P3. (a) Fas-mediated apoptosis was induced in T-cell blasts from ALPS-FASLG patients (P1 $\bigcirc$ and P3 $\bigcirc$ ), two healthy controls $(\square)(\Delta)$ and an ALPS-FAS patient $(\nabla)$, using different concentrations of anti-Fas mAb. (b) phytohemagglutinin (PHA)-mediated AICD was induced in T-cell blasts from controls, ALPSFASLG and ALPS-FAS patients using different concentrations of PHA. (c) Apoptosis and (d) loss of mitochondrial membrane potential were assessed in EBV-transformed B-cells from two controls $(\square)$ and ALPS-FASLG patients (P1 and P3 $\square$ ) using PHA, ionomycin (Ion) + PMA and anti-Fas mAb $(500 \mathrm{ng} / \mathrm{ml})$. Columns show the mean \pm SD of three independent experiments. Statistical comparisons were performed with unpaired Student's $t$-tests, with significance indicated by asterisks $\left({ }^{*} P<0.05,{ }^{* *} P<0.01\right)$, and ns, no statistical significance.

B cells. We did not observe significant differences between the patients and controls in Fas-mediated apoptosis using Apo1.3, but when B-cell lines were re-stimulated with PHA or with ionomycin + PMA, a partial reduction in AICD was also observed in P1 and P3 (Figure 3c,d). The expression of FasL was barely detectable in the EBV-transformed cell lines from the patients and controls (data not shown); however, the AICD defect was partial in ALPS-FASLG patients. These results could indicate that a Fas-FasL-independent pathway accounts for the partial cell death of FasL-deficient EBV-transformed cell lines from the patients, as has been previously described for FasLdeficient T-cells (results shown above and in refs. ${ }^{12,13}$ ). 


\section{DISCUSSION}

The present work describes two brothers with a novel homozygous FASLG mutation $(605 \mathrm{G}>\mathrm{C})$, affecting the extracellular domain of the FASLG gene and causing a severe form of ALPS. The absence of clinical symptoms in their heterozygous parents and brother suggests autosomal recessive inheritance of this Fas ligand deficiency (see Supplementary Figure S1b online). Fas/FasL-mediated cytotoxicity was severely impaired in P1, probably due to the mutation found in FASLG giving rise to a nonfunctional protein. Additionally, expression of the FASLG gene and corresponding protein was conserved, but sFasL was not be detected, suggesting that the FASLG mutation in these patients inhibits shedding of the sFasL protein (Figures 1 and 2).

The results confirm a partial reduction in AICD in both T-cell blasts and EBV-transformed B-cell lines (Figure 3), demonstrating that the impact of the mutations on AICD is very similar and showing that other pathways are acting in AICD induction, as previously described in other ALPSFASLG and ALPS-FAS patients $(12,13,17,18)$. It is remarkable to observe this difference in AICD in EBV-transformed B-cell lines, given that the percentage of cells that express FasL is low (no more than 10\%, data not shown) both in B-cells from the controls and from the patients. This could indicate that the main mechanism of Fas/FasL-mediated AICD in these cells, as observed previously by our group in human T-cell blasts (19), is mediated by the release of FasL on the surface of exosomes, inducing not only the suicide of the same exosome-releasing cells, but also the fratricide of neighboring Fas-expressing cells.

It is likely that the differential impact of FAS and FASLG mutations on AICD is attributable to this type of cell death being conserved in heterozygous ALPS-FAS patients, while it is partially impaired in homozygous ALPS-FAS and ALPSFASLG patients $(12,17,18)$. The complete absence of Fas signaling in the latter groups, patients with the homozygous forms of the syndrome, causes a defect in AICD that can be detected in vitro. However, in heterozygous ALPS-FAS patients, it seems that other Fas-independent mechanisms are playing a role in AICD and able compensate for the defect, no differences being detected in vitro.

One of these Fas-independent mechanisms could be the perforin/granzyme pathway, especially relevant for $\mathrm{CD}^{+} \mathrm{T}$-cells, the role of this pathway to AICD becoming relatively more important in the absence of a contribution from the Fas/FasL pathway (17). In spite of this possible partial compensation, the absence of Fas/FasL-mediated regulation results in severe disease, especially massive lymphoproliferation and an increased risk of lymphoma. In fact, we report here the development of a T-cell lymphoma by P3 $(12,18)$. To our knowledge, this is the first reported case of lymphoma in a patient with ALPS-FASLG. The risk of Hodgkin's and non-Hodgkin's lymphomas in families with ALPS-FAS syndrome has been estimated to be respectively 149 times and 61 times greater than expected, with the majority of lymphomas being of B-cell lineage (89\%) (5).

In summary, we describe a new case of ALPS-FASLG with a severe clinical phenotype, in addition to alterations in cytotoxicity and apoptosis. Although FasL deficiency is rare, this case represents the sixth patient described and the fourth with autosomal recessive inheritance (see Supplementary Table S1 online). Additionally, this study is the first to demonstrate defective AICD in EBV-transformed B-cells from ALPS-FASLG patients, results suggesting that the link between the partial defect in AICD in T- and B-cells in ALPS-FASLG patients and lymphoma merits further investigation.

\section{METHODS}

\section{Patients}

The study was approved by the clinical ethics committee of Hospital Universitario 12 de Octubre (Spain). Blood samples were obtained from the patients, their relatives, and healthy controls after they had given written informed consent in agreement with the principles of the Declaration of Helsinki.

\section{ALPS Biomarkers}

Proportions of DN T cells were determined in total blood samples by flow cytometry using a Navios Cytometer (Beckman Coulter, Madrid, Spain). Mouse anti-human monoclonal antibodies were used to identify this population: anti-TCR $\alpha \beta-F I T C$, anti-TCR $\gamma \delta$-PE, antiCD3-PerCP5.5 (BD Biosciences, Madrid, Spain), anti-CD8-PeCy7, anti-CD4-APC, and anti-CD45-APC-Alexa Fluor 750 (Beckman Coulter). Activated T-cells and B-cell phenotype were also tested in the ALPS-FASLG patient. Mouse anti-human monoclonal antibodies were used to identify these populations: anti-HLA-DR-PE, antiCD3-PerCP5.5, anti-IgD-PE (BD Biosciences), anti-CD19-FITC, and anti-CD27-PC5 (Beckman Coulter).

Plasma levels of IL-10 (Bender MedSystems, LabClinics, Madrid, Spain), sCD25 and sFasL (R\&D Systems, Vitro, Madrid, Spain) and vitamin B12 (Beckman Coulter) were measured in duplicate by enzyme-linked immunosorbent assay. Serum immunoglobulin levels (IgG, IgA, IgM) were measured by nephelometry (Beckman Coulter).

\section{Cells and Cell Culture}

Human peripheral-blood mononuclear cells were obtained from the blood of patients and healthy donors by Ficoll separation. T-cell blasts were generated from these cells by PHA stimulation $(20 \mathrm{ng} / \mathrm{ml}$ ) (SigmaAldrich, Madrid, Spain) in the presence of IL-2 (100 IU/ml) (Roche, Madrid, Spain), as described elsewhere (12). EBV-transformed B-cell lines derived from patients and normal donors were maintained in RPMI 1640 with $20 \%$ fetal calf serum, 2 mmol/l L-glutamine, penicillin $100 \mathrm{U} / \mathrm{ml}$ and $100 \mu \mathrm{g} / \mathrm{ml}$ streptomycin (Sigma-Aldrich) at $37^{\circ} \mathrm{C}$ in a humidified 5\% $\mathrm{CO}_{2}$ incubator. Jurkat cells (clone E6-1, derived from a T-cell acute leukemia, ATCCTIB-152) and HEK-293T cells (derived from a human embryonic kidney cell line, ATCCCRL-3216) were also used for cytotoxicity experiments.

\section{Molecular Genetics}

Mutation analysis of FAS and FASL genes was performed as described elsewhere (12).

\section{Cytotoxicity Assays}

Carboxyfluorescein succinimidyl ester (CFSE) staining of Jurkat, L1210 and L1210.Fas cells allows us to gate target cells in flow cytometry analysis and quantify cell death using 7-aminoactinomycin D (7AAD) staining exclusively in the target cell population. For FasL-induced cytolysis, 6-d-old T-cell blasts from P1, P3, and two healthy controls were tested for toxicity to Jurkat cells. We used an effector:target ratio of 2:1 in an overnight cytotoxicity assay and the results represent the percentage of Jurkat CFSE+ (Life Technologies, Madrid, Spain) cells positive for 7AAD staining (Immunostep, Salamanca, Spain).

Constructs were created and transfection was performed as described elsewhere (12). Control H293T cells, or H293T cells transfected with either the wild type or the mutant FasL constructs, were also used as effectors in cytotoxicity assays. Transfection efficiency, based on microscopic evaluation of DsRed2 expression, varied from 25 to $30 \%$. No significant differences in transfection efficiency 
were found between the wild-type and the mutant construct. Cells were tested at different effector:target ratios against CFSE-stained Fas-transfected murine L1210 cells (L1210.Fas) or against CFSEuntransfected L1210 cells negative for Fas expression (L1210) in 6-h assays. The results represent the percentage of L1210.Fas- or L1210CFSE+ cells positive for 7AAD staining.

Bioactive FasL is released to the supernatant by human cells in the form of whole, nonproteolyzed protein, associated with a particulate fraction characterized as exosomes that contain FasL on their surface (19). Accordingly, supernatants from the cultures indicated above were collected and tested for toxicity on L1210.Fas and L1210. Cell death was measured using annexin V-FITC staining (Immunostep).

\section{FASLG Expression}

Total RNA was isolated from T-cell blasts with the RNeasy plus mini kit (Qiagen, Madrid, Spain). One microgram of total cellular RNA was reverse transcribed using a Transcriptor First Strand cDNA Synthesis Kit (Roche). Gene expression was analyzed by real-time PCR using a TaqMan Fast Universal PCR Master Mix and Taqman probes (FASLG: Hs00181225_m1) (Life Technologies) in accordance with the manufacturer's instruction. GADPH was used as the endogenous control, and the level of expression of FASLG was quantitatively measured relative to that in three different donors without the mutation.

Cell lysates (RIPA buffer, Sigma-Aldrich; protease inhibitor cocktail, Thermo Scientific, Madrid, Spain) were prepared from T-cell blasts. Cell lysates were resolved on 4 to $20 \%$ precast polyacrylamide gel (Biorad, Madrid, Spain). Proteins were blotted on a nitrocellulose membrane and probed with anti-FasL (Clone NOK-1, BD Biosciences) or anti- $\beta$-actin (Sigma-Aldrich) antibodies.

Peripheral-blood mononuclear cells $\left(1 \times 10^{5}\right)$ from P1, P3, and a healthy control were stimulated for $72 \mathrm{~h}$ with PHA $(200 \mathrm{ng} / \mathrm{ml})$ (SigmaAldrich), plate-bound anti-CD3 $(10 \mu \mathrm{g} / \mathrm{ml})$ and soluble anti-CD28 $(2 \mu \mathrm{g} / \mathrm{ml})$ (BD Biosciences) or left unstimulated. Supernatants were collected and sFasL levels were measured in duplicate by enzymelinked immunosorbent assay (R\&D Systems, Vitro, Madrid, Spain).

To analyze FasL expression of T-cells and EBV-transformed B-cells, $1 \times 10^{5}$ cells were stained for FACS analysis with anti-human FasL-PE mAb (clone NOK-1; Miltenyi Biotec, Madrid, Spain)

\section{Apoptosis and AICD}

T-cell blasts $\left(1 \times 10^{5}\right.$ cells $)$ from P1, P3, and healthy controls were cultured with different concentrations of anti-Fas mAb $(125,250,500 \mathrm{ng} /$ ml) (Clon Apo1.3; Enzo Life Sciences, Madrid, Spain) and PHA (1.25, 2.5 , and $5 \mu \mathrm{g} / \mathrm{ml}$ ) (Sigma-Aldrich). After overnight incubation, apoptotic cells were detected by flow cytometry using annexin V-FITC.

EBV-transformed B-cells were aliquoted in triplicate into 96-well plates and cultured with PHA $(5 \mu \mathrm{g} / \mathrm{ml})$, ionomycin $(1.25 \mu \mathrm{g} / \mathrm{ml})$ plus phorbol-12-myristate-13-acetate (PMA, $100 \mathrm{ng} / \mathrm{ml}$ ) (SigmaAldrich) and anti-Fas (Apo1.3, 500 ng/ml). After 16h, apoptosis and loss of mitochondrial transmembrane potential were evaluated by flow cytometry using annexin V-FITC and DiOC2 staining (Life Technologies), respectively.

\section{SUPPLEMENTARY MATERIAL}

Supplementary material is linked to the online version of the paper at http:// www.nature.com/pr

\section{ACKNOWLEDGMENTS}

We thank Miguela Menchén, and María José Díaz-Madroñero for their technical assistance, and Ana Victoria Marin for providing supernantant of EBVproducing B95-8 cells.

\section{STATEMENT OF FINANCIAL SUPPORT}

This work was supported by grants from Fondo de Investigación Sanitaria (FIS-PI11/1591) to L.M.A., Fundación Mutua Madrileña (FMM-2011/0090) to E.P.A. and Ministerio de Economía y Competitividad (SAF2010-15341) to A.A. The project has been co-financed with FEDER funds.
Disclosures: The authors declare that they have no conflicts of interest.

\section{REFERENCES}

1. Canale VC, Smith $\mathrm{CH}$. Chronic lymphadenopathy simulating malignant lymphoma. J Pediatr 1967;70:891-9.

2. Fisher GH, Rosenberg FJ, Straus SE, et al. Dominant interfering Fas gene mutations impair apoptosis in a human autoimmune lymphoproliferative syndrome. Cell 1995;81:935-46.

3. Rieux-Laucat F, Le Deist F, Hivroz C, et al. Mutations in Fas associated with human lymphoproliferative syndrome and autoimmunity. Science 1995;268:1347-9.

4. Neven B, Magerus-Chatinet A, Florkin B, et al. A survey of 90 patients with autoimmune lymphoproliferative syndrome related to TNFRSF6 mutation. Blood 2011;118:4798-807.

5. Price S, Shaw PA, Seitz A, et al. Natural history of autoimmune lymphoproliferative syndrome associated with FAS gene mutations. Blood 2014;123:1989-99.

6. Magerus-Chatinet A, Stolzenberg MC, Loffredo MS, et al. FAS-L, IL-10, and double-negative CD4- CD8- TCR alpha/beta+ T cells are reliable markers of autoimmune lymphoproliferative syndrome (ALPS) associated with FAS loss of function. Blood 2009;113:3027-30.

7. Caminha I, Fleisher TA, Hornung RL, et al. Using biomarkers to predict the presence of FAS mutations in patients with features of the autoimmune lymphoproliferative syndrome. J Allergy Clin Immunol 2010;125: 946-949.e6.

8. Holzelova E, Vonarbourg C, Stolzenberg MC, et al. Autoimmune lymphoproliferative syndrome with somatic Fas mutations. N Engl J Med 2004;351:1409-18.

9. Magerus-Chatinet A, Neven B, Stolzenberg MC, et al. Onset of autoimmune lymphoproliferative syndrome (ALPS) in humans as a consequence of genetic defect accumulation. J Clin Invest 2011;121:106-12.

10. Wu J, Wilson J, He J, Xiang L, Schur PH, Mountz JD. Fas ligand mutation in a patient with systemic lupus erythematosus and lymphoproliferative disease. J Clin Invest 1996;98:1107-13.

11. Bi LL, Pan G, Atkinson TP, et al. Dominant inhibition of Fas ligand-mediated apoptosis due to a heterozygous mutation associated with autoimmune lymphoproliferative syndrome (ALPS) Type Ib. BMC Med Genet 2007;8:41.

12. Del-Rey M, Ruiz-Contreras J, Bosque A, et al. A homozygous Fas ligand gene mutation in a patient causes a new type of autoimmune lymphoproliferative syndrome. Blood 2006;108:1306-12.

13. Magerus-Chatinet A, Stolzenberg MC, Lanzarotti N, et al. Autoimmune lymphoproliferative syndrome caused by a homozygous null FAS ligand (FASLG) mutation. J Allergy Clin Immunol 2013;131:486-90.

14. Nabhani S, Hönscheid A, Oommen PT, et al. A novel homozygous Fas ligand mutation leads to early protein truncation, abrogation of death receptor and reverse signaling and a severe form of the autoimmune lymphoproliferative syndrome. Clin Immunol 2014;155:231-7.

15. Oliveira JB, Bleesing JJ, Dianzani U, et al. Revised diagnostic criteria and classification for the autoimmune lymphoproliferative syndrome (ALPS): report from the 2009 NIH International Workshop. Blood 2010;116: e35-40.

16. Brenner D, Krammer PH, Arnold R. Concepts of activated T cell death. Crit Rev Oncol Hematol 2008;66:52-64.

17. Mateo V, Ménager M, de Saint-Basile G, et al. Perforin-dependent apoptosis functionally compensates Fas deficiency in activation-induced cell death of human T lymphocytes. Blood 2007;110:4285-92.

18. Bosque A, Aguiló JI, Alava MA, et al. The induction of Bim expression in human T-cell blasts is dependent on nonapoptotic Fas/CD95 signaling. Blood 2007;109:1627-35.

19. Martínez-Lorenzo MJ, Anel A, Gamen S, et al. Activated human T cells release bioactive Fas ligand and APO2 ligand in microvesicles. J Immunol 1999;163:1274-81. 